\title{
Association Between Serum Albumin Levels and Diabetic Peripheral Neuropathy Among Patients with Type 2 Diabetes: Effect Modification of Body Mass Index
}

\author{
Ying $\mathrm{Hu}^{1-3}$, Jiancheng Wang ${ }^{1-3}$, Sha Zeng ${ }^{1-3}$, Mengxia Chen ${ }^{1-3}$, Guilin Zou ${ }^{1-3}$, Yuxia $\mathrm{Li}^{1-3}$, \\ Lingyan $\mathrm{Zhu}^{\mathrm{l}-3}$, Jixiong $\mathrm{Xu}^{\mathrm{l}-3}$
}

'Department of Endocrinology and Metabolism, First Affiliated Hospital of Nanchang University, Nanchang, Jiangxi, 330006, People's Republic of China; ${ }^{2}$ Jiangxi Clinical Research Center for Endocrine and Metabolic Disease, Nanchang, Jiangxi, 330006, People's Republic of China; ${ }^{3}$ Jiangxi Branch of National Clinical Research Center for Metabolic Disease, Nanchang, Jlangxi, 330006, People's Republic of China

Correspondence: Jixiong Xu; Jiancheng Wang, Department of Endocrinology and Metabolism, First Affiliated Hospital of Nanchang University, No. I7, Yongwaizheng Street, Nanchang, Jiangxi, 330006, People's Republic of China, Tel/Fax +86-79I-86836960, Email Jixiong.Xu@ncu.edu.cn; jasonwong2015@I63.com

\begin{abstract}
Background: The role of serum albumin in the risk of diabetic peripheral neuropathy (DPN) remains unclear. This study aimed to explore the relationship between serum albumin level and DPN, and to examine any possible effect modifiers among patients with type 2 diabetes mellitus.
\end{abstract}

Methods: This cross-sectional study was conducted in Jiangxi, China, from May 2012 to December 2014. Serum albumin levels were determined in 523 subjects, and the association between serum albumin level and DPN was evaluated using linear regression models (odds ratio [OR] and corresponding 95\% confidence interval [CI]).

Results: There was a significant inverse association between serum albumin level (per $1 \mathrm{~g} / \mathrm{L}$ increment, OR 0.95 [95\% CI 0.92-0.98]) and the risk of DPN. Accordingly, when serum albumin was assessed as quartiles, a significantly lower risk of DPN was found in participants in quartile 4 (OR 0.49 [95\% CI 0.25-0.95]), compared with those in quartile 1. Consistently, higher albumin levels ( $\geq 35 \mathrm{~g} /$ L) were associated with decreased odds for DPN (OR 0.36 [95\% CI 0.17-0.74]) compared with lower levels. Furthermore, the albumin-DPN association was significantly stronger in patients with a relatively high body mass index (BMI; $\geq 24 \mathrm{~kg} / \mathrm{m}^{2} ; \mathrm{OR} 0.91$ [95\% CI 0.85-0.98]) than in those with a low BMI $\left(<24 \mathrm{~kg} / \mathrm{m}^{2}\right.$; OR 0.99 [95\% CI 0.94-1.04]; $P$ for interaction $\left.=0.042\right)$.

Conclusion: These data suggest that serum albumin level could be a novel risk factor for DPN among patients with type 2 diabetes and relatively high BMI $\left(>24 \mathrm{~kg} / \mathrm{m}^{2}\right)$.

Keywords: serum albumin, BMI, diabetic peripheral neuropathy, type 2 diabetes mellitus

\section{Introduction}

Diabetic peripheral neuropathy (DPN) is the most frequent long-term complication among patients with type 2 diabetes mellitus (T2DM), resulting in peripheral nerve dysfunction accompanied by the typical characteristics of pain, dysesthesia, paresthesia, and numbness. ${ }^{1,2}$ It affects approximately $50 \%$ of patients over the course of disease, with high morbidity and mortality. ${ }^{3}$ Therefore, it is essential to identify and treat potential risk factors for the occurrence of DPN to improve prevention and reduce its significant disease-related burden.

Albumin is the most abundant serum protein and is exclusively synthesized in the liver and subsequently secreted into the plasma, entering both the intra- and extravascular spaces. ${ }^{4}$ Serum albumin has often been identified as a marker of nutritional status (malnutrition/overnutrition) and inflammation; ${ }^{5}$ however, its role as a potential endogenous antioxidant agent under physiological conditions is being increasingly recognized. ${ }^{6}$ Accordingly, previous studies have demonstrated 
the obvious protective influence of serum albumin in many diseases, such as myocardial infarction and cardiovascular diseases. $^{7-9}$ Some reports have also suggested that relatively low serum albumin levels in patients who experienced acute ischemic stroke increased the risk of poor outcome or death. ${ }^{10}$ In addition, we previously reported that elevated albumin levels protect against the progression of chronic kidney disease. ${ }^{11}$

Potential risk factors for DPN need to be identified, despite their complexity and difficulty. DPN can be affected by many factors, such as hyperglycemia, age, hypertension, smoking status, and hyperlipidemia. ${ }^{12-15}$ However, the cause of DPN remains to be comprehensively elucidated. Patients with diabetes, especially elderly individuals with malnutrition, often experience peripheral neuropathy. ${ }^{16,17}$ Based on the close connection between hypoproteinemia and malnutrition, ${ }^{5,18}$ we hypothesized that serum albumin levels could be used as an early predictor of DPN. However, to the best of our knowledge, only two studies have examined the association between serum albumin concentration and DPN among patients with T2DM. ${ }^{19,20}$ Furthermore, none of the previous studies has comprehensively investigated modifiers in the association between serum albumin and DPN. Therefore, the objective of the present study was to evaluate the association between serum albumin levels and the prevalence of DPN among patients with T2DM. We also assessed whether this association persisted when other potential confounders were considered.

\section{Materials and Methods}

\section{Subjects and Study Design}

Subjects included in this study were patients with T2DM routinely hospitalized in the Department of Endocrinology and Metabolism at the First Affiliated Hospital of Nanchang University (Nanchang, China) between May 2012 and December 2014. Inclusion criteria were as follows: age $\geq 18$ years; diagnosis of T2DM; and voluntarily participation and provision of informed written consent at admission. Patients with typical clinical features of type 1 diabetes, those with another specific form of diabetes, such as diabetes secondary to chronic pancreatitis or steroid treatment, those with acute myocardial infarction, heart failure, or cerebrovascular disease, kidney disease, such as urolithiasis, cancer, infection, acute diabetic complications, and other severe circulatory, respiratory, or digestive system diseases were excluded. T2DM was defined as patients with self-reported, physician-diagnosed diabetes, those taking anti-diabetic medications, or a fasting glucose level $\geq 7.0 \mathrm{mmol} / \mathrm{L}$ according to criteria from the World Health Organization. ${ }^{21} \mathrm{~A}$ total of 523 patients were enrolled, and hospitalization data from all subjects were retrospectively analyzed.

\section{DPN}

Diagnostic criteria for DPN included the following: among patients with clinical symptoms (pain, numbness, paresthesia), any one of five examinations (ankle reflex, acupuncture pain, vibration, pressure, and temperature) being abnormal according to the patient's diagnosis of neuropathy during or after diabetes; in the absence of clinical symptoms, 2 of 5 abnormalities could be diagnosed with DPN; and abnormalities revealed on nerve conduction study (ApS, Alpine Biomed, Skovlunde, Denmark), which included motor nerve conduction studies (median, ulnar, tibial, and common peroneal nerves) and sensory nerve conduction studies (median, ulnar, superficial peroneal, and sural nerves). Distal motor latency, motor nerve conduction velocity, compound muscle action potential wave amplitude, distal sensory latency, sensory nerve conduction velocity, and sensory nerve action potential wave amplitude were measured.

\section{Collection of Demographic Information, and Medical and Laboratory Data}

For this hospital-based cross-sectional study, all patients underwent standardized clinical and laboratory evaluations. Basic demographic data, including age, sex, height, weight, smoking status, systolic blood pressure (SBP), and diastolic blood pressure, were collected from medical records. Weight and height were determined in subjects wearing light clothing and no shoes by an ultrasonic instrument (Omron HNH-318, Japan). Body mass index (BMI) was calculated by dividing weight in kilograms by height in meters squared $\left(\mathrm{kg} / \mathrm{m}^{2}\right)$. Blood and urine samples collected on admission were analyzed during the study period. Fasting blood samples were collected to measure fasting plasma glucose (FPG), total cholesterol (TC), low-density lipoprotein cholesterol (LDL-C), high-density lipoprotein cholesterol, triglycerides, and 
albumin levels and were analyzed using an automated chemistry analyzer (AU5421, Olympus, Shizuoka, Japan). Glycated hemoglobin (HbA1c) content was measured using a glycated hemoglobin analyzer (D-10, Bio-Rad, Hercules, CA, USA).

\section{Statistical Analysis}

Continuous variables were analyzed using the $t$-test and expressed as mean \pm standard deviation (SD). For categorical variables, the chi-square test or Fisher's exact test was used, and data are expressed as percentage. The association between serum albumin and DPN was evaluated using linear regression models (odds ratio [OR] and corresponding 95\% confidence interval [CI]), with and without adjustments for age, sex, BMI, FPG, HbAlc, TC, and LDL-C. In addition, possible modifications of the association between serum albumin (per $1 \mathrm{~g} / \mathrm{L}$ increment) and DPN were assessed for the following variables: age ( $<60$ versus [vs] $\geq 60$ years), sex, BMI $\left(<24 \mathrm{vs} \geq 24 \mathrm{~kg} / \mathrm{m}^{2}\right), \mathrm{TC}(<5.2 \mathrm{vs} \geq 5.2 \mathrm{mmol} / \mathrm{L}), \mathrm{FPG}$ $(<6.1 \mathrm{vs} \geq 6.1 \mathrm{mmol} / \mathrm{L})$, and $\mathrm{HbAlc}(<6.5 \% \mathrm{vs} \geq 6.5 \%)$. Heterogeneity across subgroups was assessed using linear regression models and presented in forest plots, and interactions between subgroups and serum albumin concentrations were examined using likelihood ratio testing.

Differences with a two-tailed $P$-value of $<0.05$ were considered to be statistically significant in all analyses. R software (http://www.R-project.org) was used for all statistical analyses.

\section{Results}

\section{Clinical Characteristics of the Participants}

A total of 523 patients with T2DM were enrolled in the present study, including 403 in the DPN group and 120 in the non-DPN group. The demographic and clinical characteristics of the DPN and non-DPN groups are summarized in Table 1 . The mean age was $59.9 \pm 10.7$ years, and $52.4 \%$ of the patients were male. The mean concentration of serum albumin was $39.9 \pm 7.2 \mathrm{~g} / \mathrm{L}$. Patients with DPN were older, and exhibited greater FPG and LDL-C, and lower albumin levels.

Table I Characteristics of the Study Participants Without and with Diabetic Peripheral Neuropathy*

\begin{tabular}{|c|c|c|c|c|}
\hline Characteristics & Total & Without DPN & With DPN & $P$ value* \\
\hline No. of participants & 523 & 120 & 403 & \\
\hline Age, year & $59.3(10.7)$ & $56.2(11.0)$ & $60.2(10.4)$ & $<0.001$ \\
\hline Male, No. (\%) & $274(52.4)$ & $69(57.5)$ & $205(50.9)$ & 0.202 \\
\hline $\mathrm{BMI}, \mathrm{kg} / \mathrm{m}^{2}$ & $24.1(3.8)$ & $24.1(4.4)$ & $24.1(3.6)$ & 0.915 \\
\hline Current smoker, No. (\%) & $67(13.6)$ & $13(1 \mid .4)$ & $54(14.3)$ & 0.432 \\
\hline \multicolumn{5}{|l|}{ Blood pressure, $\mathrm{mmHg}$} \\
\hline Systolic BP at baseline & $134.7(19.0)$ & I3I.4 (I7.7) & $135.8(19.2)$ & 0.029 \\
\hline Diastolic BP at baseline & $83.2(11.5)$ & $82.8(10.9)$ & $83.3(11.7)$ & 0.694 \\
\hline NRF2 polymorphisms & & & & 0.090 \\
\hline $\mathrm{CC}$ & $228(43.6)$ & $46(38.3)$ & $182(45.2)$ & \\
\hline$A C$ & $246(47.0)$ & $57(47.5)$ & $189(46.9)$ & \\
\hline AA & $49(9.4)$ & $17(14.2)$ & $32(7.9)$ & \\
\hline \multicolumn{5}{|l|}{ Laboratory results } \\
\hline Fasting glucose, $\mathrm{mmol} / \mathrm{L}$ & $8.4(4.3)$ & $7.4(3.6)$ & $8.5(4.4)$ & 0.010 \\
\hline HbAlc (\%) & $8.6(2.3)$ & $8.3(2.3)$ & $8.7(2.4)$ & 0.091 \\
\hline Total cholesterol, $\mathrm{mmol} / \mathrm{L}$ & $4.3(1.2)$ & $4.2(1.4)$ & $4.4(1.1)$ & 0.083 \\
\hline Triglycerides, mmol/L & I.6 (I.5) & $1.6(1.7)$ & $1.5(1.4)$ & 0.494 \\
\hline LDL-C, mmol/L & $2.6(0.9)$ & $2.5(0.8)$ & $2.7(0.9)$ & 0.039 \\
\hline HDL-C, mmol/L & I.3 (4.7) & $1.2(0.5)$ & $1.4(5.3)$ & 0.663 \\
\hline Serum albumin, $g / L$ & $39.9(7.2)$ & $42.1(7.5)$ & $39.3(7.0)$ & $<0.001$ \\
\hline
\end{tabular}

Note: *Continuous variables are presented as mean (standard deviation), categorical variables are presented as numbers (percentages). Abbreviations: DPN, diabetic peripheral neuropathy; BP, blood pressure; BMI, body mass index; LDL-C, low-density lipoprotein cholesterol; HDL-C, highdensity lipoprotein cholesterol. 
Table 2 Characteristics of the Study Participants Stratified by Quintiles of Serum Albumin*

\begin{tabular}{|c|c|c|c|c|c|}
\hline Characteristics & QI & Q2 & Q3 & Q4 & $P$ value* \\
\hline No. of participants & 128 & 133 & $13 \mid$ & 131 & \\
\hline Age, year & $59.6(10.7)$ & $62.6(9.9)$ & $58.6(10.5)$ & $57.0(1 \mathrm{I} .1)$ & $<0.001$ \\
\hline Male, No. (\%) & $7 \mid(50.4)$ & $70(50.7)$ & $76(48.7)$ & $88(60.3)$ & 0.181 \\
\hline $\mathrm{BMI}, \mathrm{kg} / \mathrm{m}^{2}$ & $23.9(3.1)$ & $23.5(4.8)$ & $24.7(4.1)$ & $24.6(3.7)$ & 0.134 \\
\hline Current smoker, No. (\%) & $23(17.4)$ & II (8.I) & $16(11.0)$ & $12(16.1)$ & 0.081 \\
\hline \multicolumn{6}{|l|}{ NRF2 polymorphisms } \\
\hline $\mathrm{CC}$ & $66(46.8)$ & $51(37.0)$ & $71(45.5)$ & $66(45.2)$ & 0.560 \\
\hline$A C$ & $63(44.7)$ & $76(55.1)$ & $69(44.2)$ & $66(45.2)$ & \\
\hline $\mathrm{AA}$ & $12(8.5)$ & II (8.0) & $16(10.3)$ & $14(9.6)$ & \\
\hline \multicolumn{6}{|l|}{ Blood pressure, $\mathrm{mmHg}$} \\
\hline Systolic BP at baseline & I34.6 (22.2) & $135.2(19.1)$ & I34.6 (I8.9) & I35.7 (I5.9) & 0.962 \\
\hline Diastolic BP at baseline & $81.2(13.9)$ & $82.9(I 1.0)$ & $82.6(10.7)$ & $85.1(11.8)$ & 0.052 \\
\hline \multicolumn{6}{|l|}{ Laboratory results } \\
\hline Fasting glucose, $\mathrm{mmol} / \mathrm{L}$ & $9.6(5.9)$ & $8.5(3.5)$ & $8.0(4.0)$ & $7.4(3.2)$ & $<0.001$ \\
\hline $\mathrm{HbAlc}(\%)$ & $9.5(3.0)$ & $8.8(2.3)$ & $8.5(2.3)$ & $7.9(1.8)$ & $<0.001$ \\
\hline Total cholesterol, $\mathrm{mmol} / \mathrm{L}$ & $3.9(1.1)$ & $4.2(0.9)$ & $4.5(1.4)$ & $4.7(\mathrm{I} .4)$ & $<0.001$ \\
\hline Triglycerides, mmol/L & I.3 (0.8) & $\mathrm{I} .4(\mathrm{I} .2)$ & $2.2(4.1)$ & $1.6(1.2)$ & 0.009 \\
\hline LDL-C, mmol/L & $2.4(1.0)$ & $2.5(0.8)$ & $2.5(0.9)$ & $2.8(1.0)$ & $<0.001$ \\
\hline $\mathrm{HDL}-\mathrm{C}, \mathrm{mmol} / \mathrm{L}$ & $\mathrm{I} .0(0.4)$ & I.I (0.4) & $1.2(0.4)$ & $1.9(8.8)$ & 0.298 \\
\hline Serum albumin, g/L & $31.2(6.2)$ & $38.3(1.0)$ & $41.6(1.0)$ & $47.3(5.8)$ & $<0.001$ \\
\hline
\end{tabular}

Note: *Continuous variables are presented as mean (standard deviation), categorical variables are presented as numbers (percentages).

Abbreviations: BP, blood pressure; BMI, body mass index; LDL-C, low-density lipoprotein cholesterol; HDL-C, high-density lipoprotein cholesterol.

Patients were divided according to quartiles of serum albumin levels. Higher serum albumin levels were associated with decreased FPG and HbA1c levels, and decreased levels of TC and LDL-C (Table 2).

\section{Association Between Serum Albumin Level and DPN}

There was a significant inverse association between serum albumin level and the prevalence of DPN (Figure 1). Each $1 \mathrm{~g} /$ $\mathrm{L}$ increase in albumin level was associated with a 5\% (OR 0.95 [95\% CI 0.92-0.98]) reduction in the adjusted risk of DPN. Consistently, when albumin was assessed as quartiles, the multivariate-adjusted ORs for participants in the highest quartile (ie, Q4, $\geq 43.5 \mathrm{~g} / \mathrm{L}$ ) of serum albumin were 0.49 (95\% CI $0.25-0.95)$ compared with those in quartile 1 . When albumin was assessed as a categorical variable based on clinical cut-off values, compared with patients with low albumin concentrations $(<35 \mathrm{~g} / \mathrm{L})$, those with normal albumin concentrations $(\geq 35 \mathrm{~g} / \mathrm{L})$ exhibited lower odds of DPN (OR 0.36 [95\% CI 0.17-0.74]) (Table 3).

\section{Stratified Analysis According to Potential Effect Modifiers}

Stratified analyses were performed to further assess the association between serum albumin levels and DPN among the various subgroups. A significant interaction was observed between albumin level and BMI and DPN $(P=0.042)$. Among patients with a high BMI $\left(\geq 24 \mathrm{~kg} / \mathrm{m}^{2}\right)$, higher albumin concentrations were significantly associated with a lower risk of DPN (per $1 \mathrm{~g} / \mathrm{L}$ increase, OR 0.91 [95\% CI 0.85-0.98]). Among patients with low BMI $\left(<24 \mathrm{~kg} / \mathrm{m}^{2}\right)$, however, higher albumin concentrations had no significant effect on DPN (OR 0.99 [95\% CI 0.94-1.04]) (Figure 2).

None of the other variables, including age, sex, TC, FPG, or HbA1c, significantly modified the association between albumin and DPN (all $P$ for interaction $>0.05$ ).

\section{Discussion}

In this study, we investigated the relationship between serum albumin level and DPN among patients with T2DM. We found an independent inverse association between serum albumin levels and the prevalence of DPN, even after 


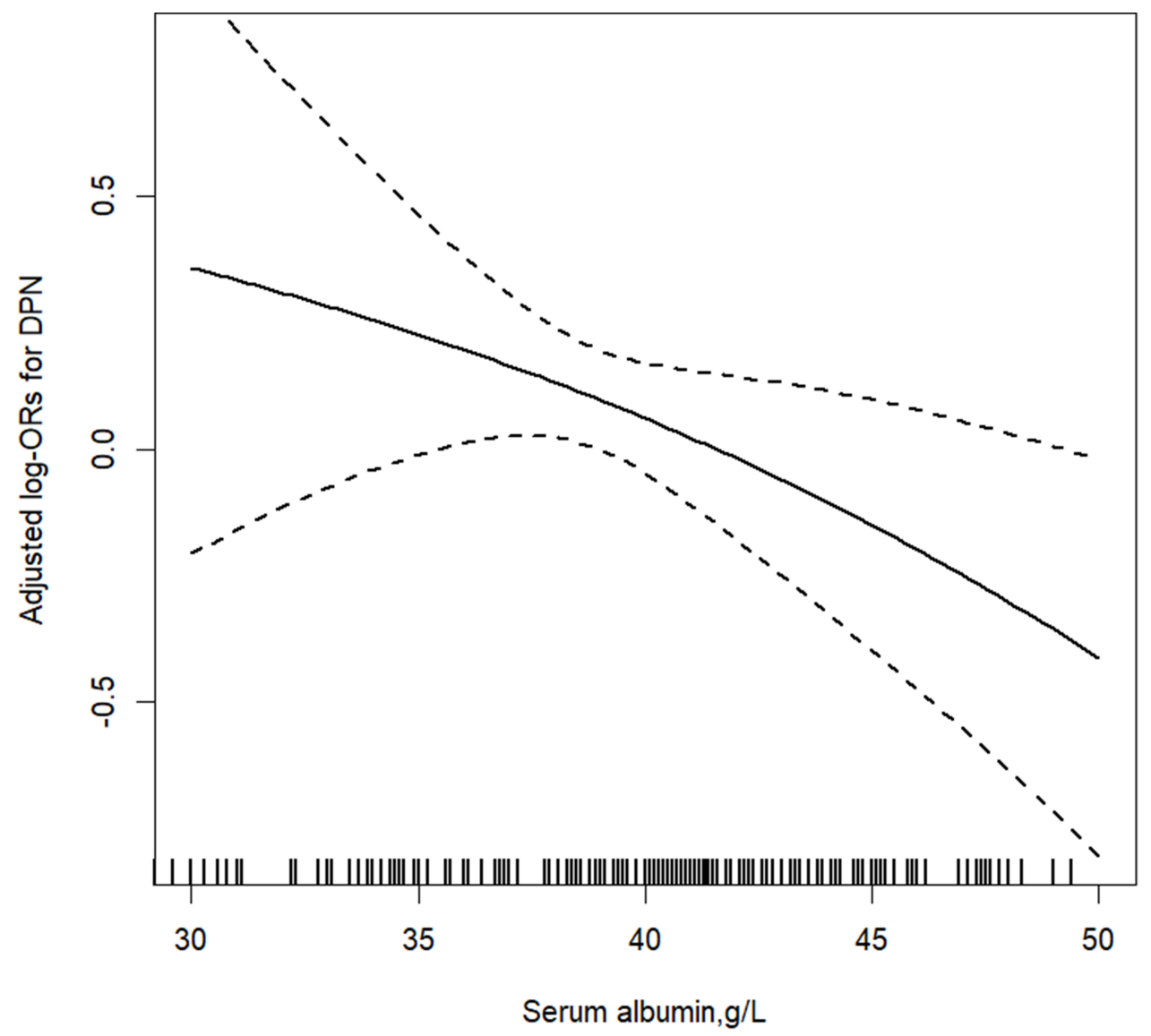

Figure I Association between serum albumin levels with diabetic peripheral neuropathy in patients with type 2 diabetes mellitus*.

Note: *Adjusted for age, sex, body mass index, fasting plasma glucose, glycated hemoglobin, total cholesterol, as well as low-density lipoprotein cholesterol.

adjustment for multiple confounding factors responsible for the pathogenesis of DPN. Moreover, our study further extends the results of previous related studies by demonstrating that the association between albumin and DPN can be modified by BMI.

Only a few studies have examined the association between serum albumin level and DPN. A previous study showed that the association between serum albumin and peripheral nerve function was significant only in patients with T2DM

Table 3 Association of Serum Albumin Levels and Diabetic Peripheral Neuropathy

\begin{tabular}{|l|c|c|c|c|}
\hline \multirow{2}{*}{ Albumin, g/L } & \multirow{2}{*}{ No. } & \multicolumn{3}{|c|}{ OR (95\% Cl) } \\
\cline { 3 - 5 } & & Unadjusted & Adjusted & P value* \\
\hline Continuous & 523 & $0.94(0.91,0.97)$ & $0.95(0.92,0.98)$ & $<0.001$ \\
Quartiles & & & & \\
Q1 (<36.9) & 128 & 1.00 & 1.00 & 0.137 \\
Q2 (36.9-40.4) & 133 & $0.65(0.34,1.24)$ & $0.61(0.31,1.17)$ & 0.051 \\
Q3 (40.4-43.5) & 131 & $0.48(0.26,0.89)$ & $0.53(0.28,1.00)$ & 0.035 \\
Q4 ( $\geq 43.5)$ & 131 & $0.43(0.23,0.79)$ & $0.49(0.25,0.95)$ & \\
P for trend & & 0.004 & 0.036 & \\
Clinical cutoff & & & & \\
$<35$ & 87 & 1.00 & $0.36(0.17,0.74)$ & 0.006 \\
$\geq 35$ & 436 & $0.34(0.16,0.70)$ & & \\
\hline
\end{tabular}

Note: *Adjusted for age, sex, body mass index, fasting plasma glucose, glycated hemoglobin, total cholesterol, as well as low-density lipoprotein cholesterol. 


\begin{tabular}{|c|c|c|c|c|c|}
\hline \multirow[b]{2}{*}{ Source } & \multicolumn{2}{|c|}{ OR (95\% CI) } & & & \multirow{2}{*}{$P$ for interaction } \\
\hline & Unadjusted & Adjusted $^{*}$ & & & \\
\hline \multicolumn{5}{|l|}{ Age, years } & \multirow{3}{*}{0.908} \\
\hline$<60$ & $0.95(0.91,0.99)$ & $0.94(0.90,0.99)$ & 0.013 & & \\
\hline$\geqslant 60$ & $0.95(0.90,0.99)$ & $0.94(0.90,1.00)$ & 0.033 & & \\
\hline \multicolumn{5}{|l|}{ Sex } & \multirow{3}{*}{0.575} \\
\hline Male & $0.94(0.90,0.98)$ & $0.94(0.90,0.98)$ & 0.006 & & \\
\hline Female & $0.95(0.91,1.00)$ & $0.96(0.91,1.00)$ & 0.065 & & \\
\hline \multicolumn{5}{|l|}{ BMI, $\mathbf{k g} / \mathbf{m i}^{2}$} & \multirow{3}{*}{0.042} \\
\hline$<24$ & $0.99(0.94,1.04)$ & $0.99(0.94,1.04)$ & 0.699 & & \\
\hline$\geqslant 24$ & $0.90(0.84,0.96)$ & $0.91(0.85,0.98)$ & 0.011 & & \\
\hline \multicolumn{5}{|c|}{ Total cholesterol, mmol/L } & \multirow{3}{*}{0.634} \\
\hline$<5.2$ & $0.94(0.90,0.97)$ & $0.95(0.92,0.98)$ & 0.005 & & \\
\hline$\geqslant 5.2$ & $0.96(0.89,1.02)$ & $0.93(0.85,1.01)$ & 0.091 & & \\
\hline \multicolumn{5}{|c|}{ Fasting glucose, mmol/L } & \multirow{3}{*}{0.848} \\
\hline$<6.1$ & $0.95(0.92,0.99)$ & $0.96(0.92,1.00)$ & 0.041 & & \\
\hline$\geqslant 6.1$ & $0.94(0.89,0.99)$ & $0.95(0.89,1.00)$ & 0.056 & 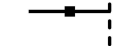 & \\
\hline \multicolumn{5}{|l|}{ HbAlC, \% } & \multirow{3}{*}{0.427} \\
\hline$<6.5$ & $0.97(0.91,1.03)$ & $0.94(0.87,1.02)$ & 0.148 & & \\
\hline \multirow[t]{2}{*}{$\geqslant 6.5$} & $0.94(0.90,0.97)$ & $0.94(0.90,0.97)$ & $<0.001$ & & \\
\hline & & & 0.8 & 0.9 & 1.1 \\
\hline
\end{tabular}

Figure 2 Association between serum albumin and diabetic peripheral neuropathy in various subgroups*.

Note: *Adjusted for age, sex, body mass index, fasting plasma glucose, glycated hemoglobin, total cholesterol, as well as low-density lipoprotein cholesterol, if not stratified.

and albuminuria. ${ }^{19}$ Additionally, in a Japanese study involving 130 patients with $\mathrm{T} 2 \mathrm{DM},{ }^{20}$ Iwasaki et al reported that serum albumin level was significantly associated with the severity of neuropathy. In the current study, we found that the association between albumin level and DPN could be modified by BMI in patients with T2DM. As such, the present study provides novel evidence supporting the neuroprotective effects of serum albumin and has shown for the first time that albumin improves DPN in patients with high BMI $\left(\geq 24 \mathrm{~kg} / \mathrm{m}^{2}\right)$.

DPN has been associated with the effects of chronic hyperglycemia on all peripheral nerves. ${ }^{22}$ Long-term exposure to hyperglycemia causes oxidative stress, which has been reported to be associated with microvascular complications in T2DM. ${ }^{23-25}$ There is accumulating evidence supporting the concept that oxidative stress is a biochemical trigger for nerve dysfunction and improved sensory nerve conduction velocity in diabetic rats. ${ }^{26,27}$ Notably, the generation of reactive oxygen species and subsequent mitochondrial and DNA damage in diabetic neuropathy cause microangiopathy, ischemia, and, ultimately, peripheral nerve malfunction. ${ }^{28}$ Accordingly, the treatment of diabetic rats with insulin or antioxidants is associated with improved nerve function. ${ }^{29}$ Therefore, the potential neuroprotective role of albumin may be related to its antioxidant properties.

Serum albumin level is widely used as an index of nutritional status. ${ }^{5}$ BMI is another important variable for assessing nutritional status. ${ }^{30}$ Therefore, it is possible that the combination of BMI and serum albumin level reflects the state of nutritional deficiency in greater detail. A prospective cohort study found that a combination of low BMI and serum albumin levels in patients with chronic kidney disease (stages 2-5) predicts a rapid decline in renal function more accurately than either factor alone. ${ }^{31}$ In addition, poor functional recovery from stroke has been noted in a population of patients with low BMI and serum albumin levels. ${ }^{32}$ These results suggest that the combination of BMI and serum albumin level can be a more robust nutritional marker. Several previous studies have investigated the relationship between BMI and DPN. ${ }^{33,34}$ Accordingly, in the current study, there was a significant inverse association between serum albumin level and the prevalence of DPN among patients with a high BMI. However, the exact mechanism(s) by which serum albumin improves DPN in patients with high BMI remains to be further investigated, and our findings and hypotheses warrant further confirmation.

The strengths of this study include the strict enrolment criteria and the ability to perform detailed, careful assessment of patients with T2DM. All staff performing vascular and foot assessments, data collection, and entry were carefully trained and accredited to ensure data quality. Second, we used subgroup analysis to identify the interaction between 
serum albumin level and BMI. To the best of our knowledge, this is the first study to demonstrate the effect of BMI on the association between serum albumin and the prevalence of DPN. Finally, we adjusted for as many confounding factors as possible. These considerations suggest that the results were reliable.

Nevertheless, our study has some limitations that should be considered when interpreting the results. First, the present investigation was cross-sectional in design, which limited our ability to infer a causal association between serum albumin levels and the risk of developing DPN. Second, the patients were admitted to university hospitals; therefore, our study results may not be applicable to the general population or patients with T2DM in primary care settings. As such, further prospective studies with larger sample sizes are required to confirm the findings of this study.

In general, we devoted little attention to low serum albumin concentration as a risk factor for DPN, although serum albumin concentration can be measured easily in the clinical laboratory and used in medical practice. Therefore, it is important to clarify the clinical significance of serum albumin levels in DPN and stratify high-risk groups for DPN according to serum albumin levels. More importantly, our findings revealed the potential of albumin as an interventional target for DPN.

\section{Conclusions}

In conclusion, our data suggest that serum albumin level was independently associated with DPN among patients with T2DM, especially among those with a high BMI $\left(\geq 24 \mathrm{~kg} / \mathrm{m}^{2}\right)$. Further studies are needed to verify whether modulating albumin levels could be beneficial to patients with DPN.

\section{Ethics Approval and Consent to Participate}

This study was approved by the ethics committee of the First Affiliated Hospital of Nanchang University (no. CDYFY2014009) and written informed consent was obtained from all individuals. This study was performed in adherence to the principles of the Declaration of Helsinki.

\section{Acknowledgments}

All authors made substantial contributions to conception and design, acquisition of data, or analysis and interpretation of data; took part in drafting the article or revising it critically for important intellectual content; agreed to submit to the current journal; gave final approval of the version to be published; and agree to be accountable for all aspects of the work.

\section{Funding}

The study was supported by funding from the following: the National Natural Science Funds of China (Nos. 81760168 and 81460018) and the National Natural Science Funds of Jiangxi Province (No. 20192BAB205031).

\section{Disclosure}

All authors declare they do not have conflicts of interest.

\section{References}

1. Polydefkis M, Griffin JW, McArthur J. New insights into diabetic polyneuropathy. JAMA. 2003;290(10):1371-1376.

2. Brownlee M. Biochemistry and molecular cell biology of diabetic complications. Nature. 2001;414(6865):813-820. doi:10.1038/414813a

3. Balcioglu AS, Muderrisoglu H. Diabetes and cardiac autonomic neuropathy: clinical manifestations, cardiovascular consequences, diagnosis and treatment. World J Diabetes. 2015;6(1):80-91. doi:10.4239/wjd.v6.i1.80

4. Barle H, Nyberg B, Essén P, et al. The synthesis rates of total liver protein and plasma albumin determined simultaneously in vivo in humans. Hepatology. 1997;25(1):154-158.

5. Fleck A, Smith G. Assessment of malnutrition in elderly patients. Lancet. 1991;337(8744):793. doi:10.1016/0140-6736(91)91412-N

6. Quinlan GJ, Martin GS, Evans TW. Albumin: biochemical properties and therapeutic potential. Hepatology. 2005;41(6):1211-1219. doi:10.1002/ hep. 20720

7. Yoshioka G, Tanaka A, Nishihira K, et al. Prognostic impact of serum albumin for developing heart failure remotely after acute myocardial infarction. Nutrients. 2020;12(9):2637. doi:10.3390/nu12092637

8. Liao LZ, Zhang SZ, Li WD, et al. Serum albumin and atrial fibrillation: insights from epidemiological and mendelian randomization studies. Eur J Epidemiol. 2020;35(2):113-122. doi:10.1007/s10654-019-00583-6

9. Arques S. Human serum albumin in cardiovascular diseases. Eur J Intern Med. 2018;52:8-12. doi:10.1016/j.ejim.2018.04.014 
10. Dziedzic T, Slowik A, Szczudlik A. Serum albumin level as a predictor of ischemic stroke outcome. Stroke. 2004;35(6):e156-8. doi:10.1161/01. STR.0000126609.18735.be

11. Jiang C, Wang B, Li Y, et al. U-shaped association between serum albumin and development of chronic kidney disease in general hypertensive patients. Clin Nutr. 2020;39(1):258-264. doi:10.1016/j.clnu.2019.02.002

12. Gregory JA, Jolivalt CG, Goor J, et al. Hypertension-induced peripheral neuropathy and the combined effects of hypertension and diabetes on nerve structure and function in rats. Acta Neuropathol. 2012;124(4):561-573. doi:10.1007/s00401-012-1012-6

13. Abdissa D, Hamba N, Kene K, et al. Prevalence and determinants of peripheral neuropathy among type 2 adult diabetes patients attending Jimma University Medical Center, Southwest Ethiopia, 2019, an institutional-based cross-sectional study. J Diabetes Res. 2020;2020:9562920.

14. Naqvi SS, Imani S, Hosseinifard H, et al. Associations of serum low-density lipoprotein and systolic blood pressure levels with type 2 diabetic patients with and without peripheral neuropathy: systemic review, meta-analysis and meta-regression analysis of observational studies. $B M C$ Endocr Disord. 2019;19(1):125. doi:10.1186/s12902-019-0453-5

15. Szwarcbard N, Villani M, Earnest A, et al. The association of smoking status with glycemic control, metabolic profile and diabetic complicationsresults of the Australian National Diabetes Audit (ANDA). J Diabetes Complications. 2020;34(9):107626. doi:10.1016/j.jdiacomp.2020.107626

16. Kuo MC, Huang JC, Wu PY, et al. Associations of small fiber neuropathy with geriatric nutritional risk index and arterial stiffness in hemodialysis. Dis Markers. 2020;2020:1694218. doi:10.1155/2020/1694218

17. Aasheim ET, Hofsø D, Hjelmesaeth J, et al. Peripheral neuropathy and severe malnutrition following duodenal switch. Obes Surg. 2008;18 (12):1640-1643. doi:10.1007/s11695-008-9539-2

18. Friedman AN, Fadem SZ. Reassessment of albumin as a nutritional marker in kidney disease. J Am Soc Nephrol. 2010;21(2):223e30. doi:10.1681/ ASN.2009020213

19. Li L, Liu B, Lu J, et al. Serum albumin is associated with peripheral nerve function in patients with type 2 diabetes. Endocrine. 2015;50 (2):397-404.

20. Iwasaki T, Togashi Y, Terauchi Y. Significant association of serum albumin with severity of retinopathy and neuropathy, in addition to that of nephropathy, in Japanese type 2 diabetic patients. Endocr J. 2008;55(2):311-316. doi:10.1507/endocrj.K07-086

21. Alberti KG, Zimmet PZ. Definition, diagnosis and classification of diabetes mellitus and its complications. Part 1: diagnosis and classification of diabetes mellitus provisional report of a WHO consultation. Diabet Med. 1998;15(7):539-553. doi:10.1002/(SICI)1096-9136(199807)15:7<539:: AID-DIA668>3.0.CO;2-S

22. Forbes JM, Cooper ME. Mechanisms of diabetic complications. Physiol Rev. 2013;93(1):137-188. doi:10.1152/physrev.00045.2011

23. Kang Q, Yang C. Oxidative stress and diabetic retinopathy: molecular mechanisms, pathogenetic role and therapeutic implications. Redox Biol. 2020;37:101799.

24. Li C, Miao X, Li F, et al. Oxidative stress-related mechanisms and antioxidant therapy in diabetic retinopathy. Oxid Med Cell Longev. 2017;2017:9702820. doi:10.1155/2017/9702820

25. Giacco F, Brownlee M, Schmidt AM. Oxidative stress and diabetic complications. Circ Res. 2010;107(9):1058-1070. doi:10.1161/ CIRCRESAHA.110.223545

26. Wang H, Ding XG, Li SW, et al. Role of oxidative stress in surgical cavernous nerve injury in a rat model. J Neurosci Res. 2015;93(6):922-929. doi:10.1002/jnr.23545

27. Sirisha A, Gaur GS, Pal P, et al. Effect of honey and insulin treatment on oxidative stress and nerve conduction in an experimental model of diabetic neuropathy Wistar rats. PLoS One. 2021;16(1):e0245395. doi:10.1371/journal.pone.0245395

28. Sifuentes-Franco S, Pacheco-Moisés FP, Rodríguez-Carrizalez AD, et al. The role of oxidative stress, mitochondrial function, and autophagy in diabetic polyneuropathy. J Diabetes Res. 2017;2017:1673081. doi:10.1155/2017/1673081

29. Dhaliwal J, Dhaliwal N, Akhtar A, et al. Beneficial effects of ferulic acid alone and in combination with insulin in streptozotocin induced diabetic neuropathy in Sprague Dawley rats. Life Sci. 2020;255:117856.

30. Casas-Vara A, Santolaria F, Fernández-Bereciartúa A, et al. The obesity paradox in elderly patients with heart failure: analysis of nutritional status. Nutrition. 2012;28(6):616-622. doi:10.1016/j.nut.2011.10.006

31. Kikuchi H, Kanda E, Mandai S, et al. Combination of low body mass index and serum albumin level is associated with chronic kidney disease progression: the chronic kidney disease-research of outcomes in treatment and epidemiology (CKD-ROUTE) study. Clin Exp Nephrol. 2017;21 (1):55-62. doi:10.1007/s10157-016-1251-2

32. Kimura Y, Yamada M, Kakehi T, et al. Combination of low body mass index and low serum albumin level leads to poor functional recovery in stroke patients. J Stroke Cerebrovasc Dis. 2017;26:448-453. doi:10.1016/j.jstrokecerebrovasdis.2016.10.008

33. Liu X, Xu Y, An M, et al. The risk factors for diabetic peripheral neuropathy: a meta-analysis. PLoS One. 2019;14(2):e0212574. doi:10.1371/ journal.pone.0212574

34. Zhang Y, Guo Y, Shen X, et al. Lower body mass index is not of more benefit for diabetic complications. J Diabetes Investig. 2019 ; $10(5)$ :1307. doi:10.1111/jdi.13003

Diabetes, Metabolic Syndrome and Obesity: Targets and Therapy

\section{Dovepress}

\section{Publish your work in this journal}

Diabetes, Metabolic Syndrome and Obesity: Targets and Therapy is an international, peer-reviewed open-access journal committed to the rapid publication of the latest laboratory and clinical findings in the fields of diabetes, metabolic syndrome and obesity research. Original research, review, case reports, hypothesis formation, expert opinion and commentaries are all considered for publication. The manuscript management system is completely online and includes a very quick and fair peer-review system, which is all easy to use. Visit http://www.dovepress. com/testimonials.php to read real quotes from published authors.

Submit your manuscript here: https://www.dovepress.com/diabetes-metabolic-syndrome-and-obesity-targets-and-therapy-journal 